\title{
ENDOSCOPIC MYRINGOPLASTY: OUR EXPERIENCE
}

\section{P. B. Kameswar Rao' ${ }^{1}$ S. Devi Prasad 2 , S. Ramesh ${ }^{3}$}

${ }^{1}$ Associate Professor, Department of ENT, GEMS, Ragolu, Srikakulam.

${ }^{2}$ Consultant, Department of ENT, Sindhura Hospital, Srikakulam.

${ }^{3}$ Assistant Professor, Department of ENT, RIMS, Srikakulam.

\section{ABSTRACT}

\section{BACKGROUND}

Myringoplasty is a commonly performed surgical procedure usually with a microscope. Endoscope is being widely used to perform many surgical procedures and now these are gaining importance in operating in cavities.

\section{AIMS}

The present study discusses our experience in performing myringoplasty using an endoscope.

\section{METHODS AND MATERIAL}

In this series 60 cases with CSOM who attended the ENT outpatient department of GEMS, Srikakulam were selected. We report the results of 60 myringoplasties performed using endoscope.

\section{RESULTS AND CONCLUSIONS}

Our study reveals that results of myringoplasty performed using endoscope are more or less similar to that of myringoplasty performed using microscope.

\section{KEYWORDS}

Myringoplasty, Endoscopic, Microscopy.

HOW TO CITE THIS ARTICLE: Rao PBK, Prasad SD, Ramesh S. Endoscopic myringoplasty: our experience. J. Evolution Med. Dent. Sci. 2016;5(39):2383-2385, DOI: 10.14260/jemds/2016/554

\section{INTRODUCTION}

Chronic suppurative otitis media has been traditionally described as a chronic inflammation of part or the entire tympanomastoid compartment comprising of Eustachian tube, the tympanic cavity, the mastoid antrum and all the pneumatized spaces of temporal bone associated with perforation of the tympanic membrane and otorrhoea. Myringoplasty is a surgical procedure performed to close tympanic membrane perforation. The operating microscope made significant advances in middle ear surgery possible for a period of almost 5 decades, but the drawback was unobservable blind angles in the middle ear cavity and a magnified image along a straight line only. ${ }^{1}$ The Endoscopes provide magnified image with good resolution and corners of middle ear cavity can be visualized with minimal effort. ${ }^{2}$ Even difficult areas in the middle ear cleft like sinus tympani can be visualized better with an endoscope when compared with microscope. ${ }^{3}$

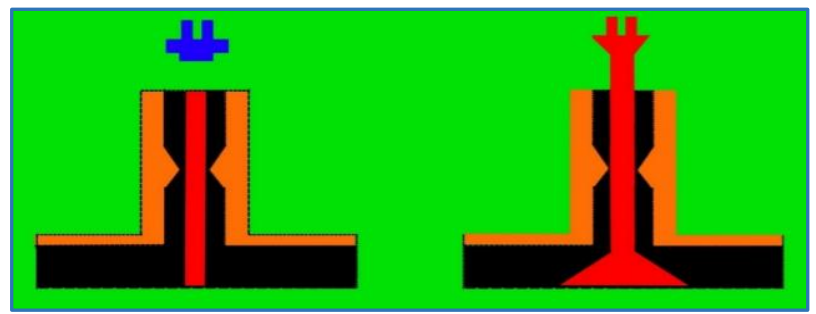

Fig. 1: Showing the Magnified Line of Vision in an Endoscope

Financial or Other, Competing Interest: None.

Submission 30-03-2016, Peer Review 25-04-2016,

Acceptance 03-05-2016, Published 16-05-2016.

Corresponding Author:

Dr. S. Ramesh,

Assistant Professor

RIMS, Srikakulam.

E-mail: rameshseepana@rediffmail.com

DOI: $10.14260 /$ jemds $/ 2016 / 554$

\section{MATERIALS AND METHODS}

In this series 60 cases with CSOM who attended the ENT Outpatient Department of GEMS, Srikakulam were selected.

1. A thorough history, clinical examination and investigations were carried out.

2. The patients who were presented with ear discharge were treated for a minimum period of 10 days and surgery was taken up after 6 weeks.

3. Pure-tone audiometry was performed for all these patients preoperatively. All of them had 30-40 dB conductive hearing loss. Post-operatively, pure-tone audiometry is performed 6 weeks after surgery.

\section{Inclusion Criteria}

1. Patients aged between $15-50$ years and both sexes were included.

2. Patients with CSOM of mucosal type having dry ear for 6 weeks.

3. Patients with demonstrable degree of conductive deafness were chosen (at least $30 \mathrm{~dB}$ pure tone average)

\section{Exclusion Criteria}

1. Patients with narrow external auditory canal.

\section{PROCEDURE}

All these patients underwent surgery under local anaesthesia. The patient is premedicated with intramuscular injections of 1 cc Fortwin and 1 cc Phenergan. The external auditory canal is then anaesthetized using $2 \%$ xylocaine mixed with 1 in 10,000 adrenaline injection at 3-o clock, 6-o clock, 9-o clock and 12-o clock positions at bony cartilaginous junction. A curvilinear incision of $3 \mathrm{~cm}$ given in the supra-auricular region and temporalis fascia graft is harvested and allowed to dry.

Step I

Freshening the margins of perforation - Margins of the perforation is freshened using sickle knife or angled pick so as 
to break the adhesions formed between the squamous margin of the ear drum with that of the middle ear mucosa. This is followed by scraping of under surface of the tympanic membrane round the perforation.

\section{Step II}

Elevation of tympano-meatal flap. Using a drum knife, a curvilinear incision is made about $4 \mathrm{~mm}$ lateral to the annulus. This incision extends between the 1-o clock, 3-o clock and 7-o clock positions in the left ear and 11-o clock, 9-o clock and 5-o clock positions in the right ear. The flap is slowly elevated away from the bone of the external canal up to the level of annulus.

\section{Step III}

Elevation of the annulus and incising the middle ear mucosa. The annulus is gradually lifted from its rim. As soon as the annulus is elevated, a sickle knife is used to incise the middle ear mucosal attachment with the tympano-meatal flap at the level of posterior spine just above the chorda.

\section{Step IV}

Freeing the tympano-meatal flap from the handle of malleus. The tympano-meatal flap is freed from the handle of malleus by sharp dissection of the middle ear mucosa with sickle knife.

\section{Step V}

Placement of graft (Underlay technique). A properly dried temporalis fascia graft of appropriate size is introduced through the ear canal. The graft is gently pushed under the tympano-meatal flap, which has been elevated. The graft is kept under the handle of malleus. The tympano-meatal flap is repositioned in such a way that it covers the free edge of the graft, which has been introduced. Success of myringoplasty was assessed both subjectively and objectively.

\section{Subjective Indicators Include}

1. Hearing improvement.

2. Absence of ear discharge.

\section{Objective Indicators are}

1. Otoendoscopic evidence of healed perforation.

2. Improvement in hearing threshold demonstrated by performing pure tone audiometry.

Success rate of endoscopic procedure was compared with that of various studies performed using microscopic approach.

\section{RESULTS}

A prospective clinical study with 60 patients undergone endoscopic myringoplasty is undertaken.

\section{Age and Sex Distribution}

The most common age group with CSOM was 21-30 years. There were $33(55 \%)$ male and 27 (45\%) female patients.

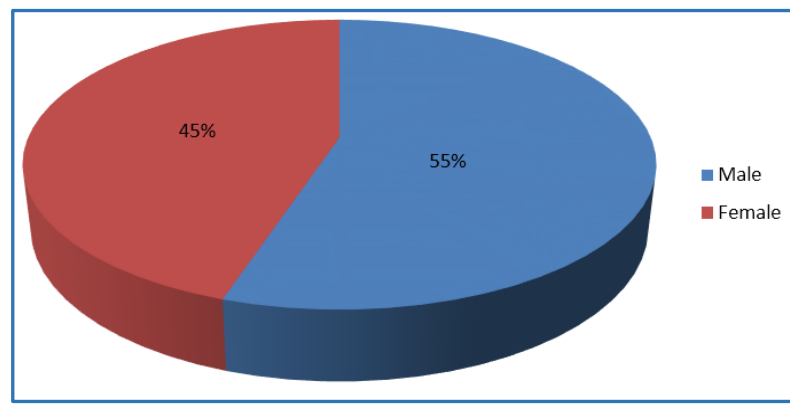

Fig. 2: Showing Sex Distribution

2. Socioeconomic Status

Most of the patients 40 (66\%) are from low socioeconomic status.

3. Third Week after Surgery

$49(81.7 \%)$ patients out of 60 had intact ear drum.

4. Sixth Week after Surgery

$53(88.3 \%)$ patients out of 60 had intact ear drum with dry ear.

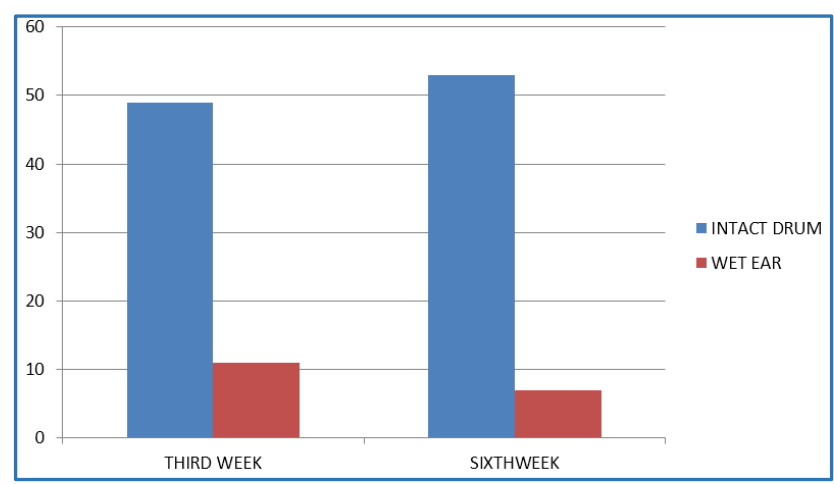

Fig. 3: Graph showing Objective Reports of a Total of 60 Patients who Underwent Endoscopic Myringoplasty

5. Hearing Improvement: Preop Audiometry showed A. $39(65 \%)$ patients had $30-35 \mathrm{~dB}$ hearing loss B. $21(35 \%)$ patients had 35-40 dB hearing loss

Postop Audiometry: 51 (85\%) patients after endoscopic myringoplasty had a pure tone hearing average of 25-30 dB. In $9(15 \%)$ patients, improvement in air conduction threshold is not seen.

\section{DISCUSSION}

Myringoplasty is defined as repair of tympanic membrane perforation. It is commonly performed surgical procedure done using microscope. Endoscopic ear surgery employs very similar techniques to those of standard microscopic ear surgery, but with different approach to the middle ear and to middle ear pathology. Endoscopes provide an excellent magnified image with good resolution, which can be achieved by just getting the endoscope closer to the field. With minimal effort corners and recesses of middle ear can be seen. Endoscopic myringoplasty fulfils the criteria of minimally invasive surgical procedure. ${ }^{4}$ Perhaps the greatest disadvantage in this technique is the one-handed nature of endoscopic ear surgery. The endoscope is held in the nondominant hand, while the opposite hand undertakes the majority of the surgery. But when analysed, the function of the 
non-dominant hand during traditional microscopic surgery is usually concerned with holding a suction and removing blood from the operative field, while the dominant hand still undertakes the majority of the delicate surgery. Given that an endoscopic approach is considerably less traumatic than a standard microscopic approach to the middle ear, there is usually far less bleeding and as a result the need for suction is reduced considerably.

A prospective clinical study done on 60 patients undergone endoscopic myringoplasty. Males had higher preponderance for CSOM, when compared to females. Prominence of males (55\%) seen in our study was also supported by Kangsanarak et al (1993), Singh and Maharaj (1993) and Sriyanon et al (1984). However, Shamboul KM (1992) reported predominance of females.

The disease was commonly seen in low and middle socioeconomic groups in $2^{\text {nd }}$ to $3^{\text {rd }}$ decade of life in our study. According to Moustafa et al (2009), patients in the first three decades of life from low socio-economic group were more commonly associated with complications, but there was no sex preponderance. ${ }^{5}$ In our study $49(81.7 \%)$ patients had intact ear drum by third week without ear discharge and by the end of sixth week $53(88.3 \%)$ had healed membrane with dry ear. Post-operatively, $51(85 \%)$ patients had a pure tone hearing average of 25-30 dB by the end of sixth week and in $9(15 \%)$ patients, improvement in air conduction threshold is not seen. Our outcomes fall within the range of successful myringoplasty rates done with microscope described in the literature as regards to graft intake (71\%-96\%).6,7 and post-operative hearing improvement (72\%-97\%).6,8,9

\section{CONCLUSION}

We conclude that the results of endoscopic myringoplasty are comparable with that of microscope myringoplasty. Even though the learning curve is a little steep, it is worthwhile exploring this option due to the obvious advantages.

\section{REFERENCES}

1. Jadav SP, Aggarwal N, Julaha M, et al. Endoscope assisted myringoplasty. Singapore medical journal 2009;50(5):510-2.

2. Patil RN. Endoscopic tympanoplasty-definitely advantageous (preliminary reports). Asian J Ear Nose Throat 2003;25:9-13.

3. Khan I, Jan AM, Shahzad F. Middle-ear reconstruction: a review of 150 cases. J Laryngol Otol 2002;116(6):435-9.

4. Karhuketo TS, Ilomaki JH, Pukakka HJ. Tympanoscopeassisted myringoplasty. ORL J Otorhinolaryngol Relat Spec 2001;63(6):353-7.

5. Moustafa BE, El Fiky, El Sharnousy MM. Complications of suppurative otiitis media: still a problem in 21st century. J Otorhinolaryngol Head Neck Surg 2009;71(2):87-92.

6. Sergi B, Galli J, De Corso E, et al. Overlay versus underlay myringoplasty: report of outcomes considering closure of perforation and hearing function. Acta Otorhinolaryngol Ital 2011;31(6):366-71.

7. Onal K, Uguz MZ, Kazikdas KC, et al. A multivariate analysis of otological, surgical and patient-related factors in determining success in myringoplasty. Clin Otol 2005;30(2):115-20.

8. Umapathy N, Dekker PJ. Myringoplasty: is it worth performing in children? Arch Otolaryngol Head Neck Surg 2003;129(10):1053-5.

9. Karela M, Berry S, Watkins A, et al. Myringoplasty: surgical outcomes and hearing improvement: is it worth performing to improve hearing? Eur Arch Otorhinolaryngol 2008;265(9):1039-42. 